\title{
Aural foreign body removal: there is no one-size-fits-all method
}

This article was published in the following Dove Press journal: Open Access Emergency Medicine

\section{Tian-Tee $\mathrm{Ng}$}

ENT Unit, Department of Surgery, Frankston Hospital. Peninsula Health, Frankston 3/99, VIC, Australia
Correspondence: Tian- $\mathrm{Tee} \mathrm{Ng}$ ENT Unit, Department of Surgery, Frankston Hospital, Peninsula Health, PO Box 52, Hastings Road, Frankston 3199, VIC, Australia

Tel +6I 0426266890

Email tntdynamites@yahoo.com
Background: Managing patients with aural foreign body (AFB) may pose a dilemma regarding which removal technique to use for different AFB types. The current study comprises a review of all the possible methods one could employ in removing AFB. My aim was to describe the best methods for different types of AFBs, complete with a description of the method and tool(s) required, and descriptions of the AFBs for which they are best used.

Materials and methods: The medical literature published between 2000 and 2016 was reviewed using Medline, Cinahl, Embase, Cochrane, PubMed, and Scopus to compile a list of all published AFB removal methods.

Results: Ten methods were identified and described, each having their own advantages for different AFBs. Patients normally permit very few attempts, so the first AFB removal attempt should ideally be the only one.

Conclusion: There is no single method guaranteed to work with all AFBs, so this report also contains a flowchart to aid deciding which technique to use.

Keywords: foreign body, ear, emergency care

\section{Introduction}

Aural foreign body (AFB) removal is a common procedure in a hospital emergency department. The range of items commonly found as AFBs and the array of different techniques to remove AFBs described in the literature do not support a straightforward decision of which technique to use for those doctors managing these patients. This was particularly apparent following a retrospective study titled "A 5 year review of aural foreign body removal in a Major Victorian hospital". ${ }^{1}$ The latter study found the most commonly found AFBs to range from insects, cotton tips, beads, ear phone/hearing aid parts, food pieces, parts of ear plugs, putty, stone, pebble, plastic bits, paper bits, rubber bits, and styrofoam balls. The current study comprises a review of the different techniques to remove AFBs, including how they may be best employed and with what types of AFBs, in order to maximize the chances of success.

\section{Materials and methods}

A literature review was conducted in March 2017 (from Jan 2000 to Dec 2016) with a search strategy aimed to identify articles describing AFB removal methods. Medline, Cinahl, Embase, Cochrane, PubMed, and Scopus were searched to compile a list of possible methods to remove AFBs, complete with a description of the method and tool(s) required, and descriptions of the AFBs for which they are best used. The key search terms used were 
"ear", "foreign body", and "removal". Articles selected were only those written in English language, and included original articles, review articles, case series, and case reports. In this collection of articles, eight were found, which described ten methods of removing AFBs. One method specifically excluded in this study involved using impression materials to remove an AFB. ${ }^{2}$ This was excluded due to anecdotal reports of dangerous use, for example, if it was accidentally used in someone with a perforated tympanic membrane (TM). There are other safer and easier methods available. Ethical approval was not obtained due to the lack of intervention arm in the study.

\section{Results}

\section{Superglue (cyanoacrylate)}

\section{Technique}

Apply superglue to the wooden end of a cotton-tipped applicator and place this end against the foreign body (FB) in the ear. After 30 seconds, remove the applicator with the adherent FB.

\section{Good for}

This is good for cooperative patients with non-graspable FB, that is, a FB that occludes so much of external auditory canal (EAC) that there is no room for an instrument to pass by.

\section{Note}

The cyanoacrylate in superglue confers its fast-acting bonding properties when it contacts a hardener such as water. Hence, if the superglue touches a damp surface such as in the ear, the glue will form a tight bond between the surfaces within seconds, thus care must be taken. ${ }^{3}$

\section{Acetone/hydrogen peroxide}

Acetone can easily be bought off-the-shelf from pharmacies.

\section{Technique}

Place warmed acetone or 3\% hydrogen peroxide into the EAC, allow the solution to sit for 10 minutes, then carefully remove and peel the FB out. A second application of acetone or hydrogen peroxide may be needed for the FB to come in one piece. The EAC should be irrigated with warmed sterile water following removal of FB with either acetone or hydrogen peroxide.

\section{Good for}

This is good for removal of superglue (cyanoacrylate) or gum.

\section{Note}

Warn patient of the risk of TM perforation if superglue accidentally adheres to the TM. ${ }^{4}$ This should not be used in patients with TM perforation because of the risk of ototoxicity to the middle and inner ear.

\section{Irrigation}

\section{Technique}

Water for irrigation must be warmed to body temperature. Flow should be brisk and aimed at the posterior-superior aspect of the EAC to flush the FB out into a dish placed below the ear. ${ }^{5}$ Recently, a pulsed flow has been shown to improve the removal success rate at low pressures known to not injure the TM. ${ }^{2,6}$

\section{Good for}

This is good for round or smooth objects to be floated out or small FBs located close to the TM or a live insect.

\section{For a live insect}

Effort should be made to kill the insect safely and quickly to provide immediate symptom relief. ${ }^{7}$ A simple and effective method is to use a $10 \mathrm{~mL}$ "water for injection" plastic ampule which has been warmed to body temperature. Gently squeeze a few drops into the EAC until the canal is full and shine a bright light into the canal. If capable, the insect will swim to the surface and can be helped out using the plastic ampule as a "life raft". If not, the insect drowns and can be more easily removed, as it is no longer moving. ${ }^{7}$

\section{Note}

Avoid using if an irrigation fluid may cause additional problems, for example, hygroscopic FBs that may swell when exposed to water or button batteries. This method is also contraindicated in those patients with a perforated TM or if their TM status is unknown.

\section{Crocodile/alligator forceps}

The crocodile/alligator forceps are shown in Figure 1.

\section{Technique}

Gently grasp the FB with crocodile forceps. It is important to refrain from clamping hard on objects that may prove fragile and can crumble, making them more difficult to remove. ${ }^{5}$

\section{Good for}

This is good for irregular or soft graspable objects.

\section{Note}

Crocodile forceps are the most common tool used for removal of AFBs by the non-otolaryngologist. ${ }^{8}$ However, they are 


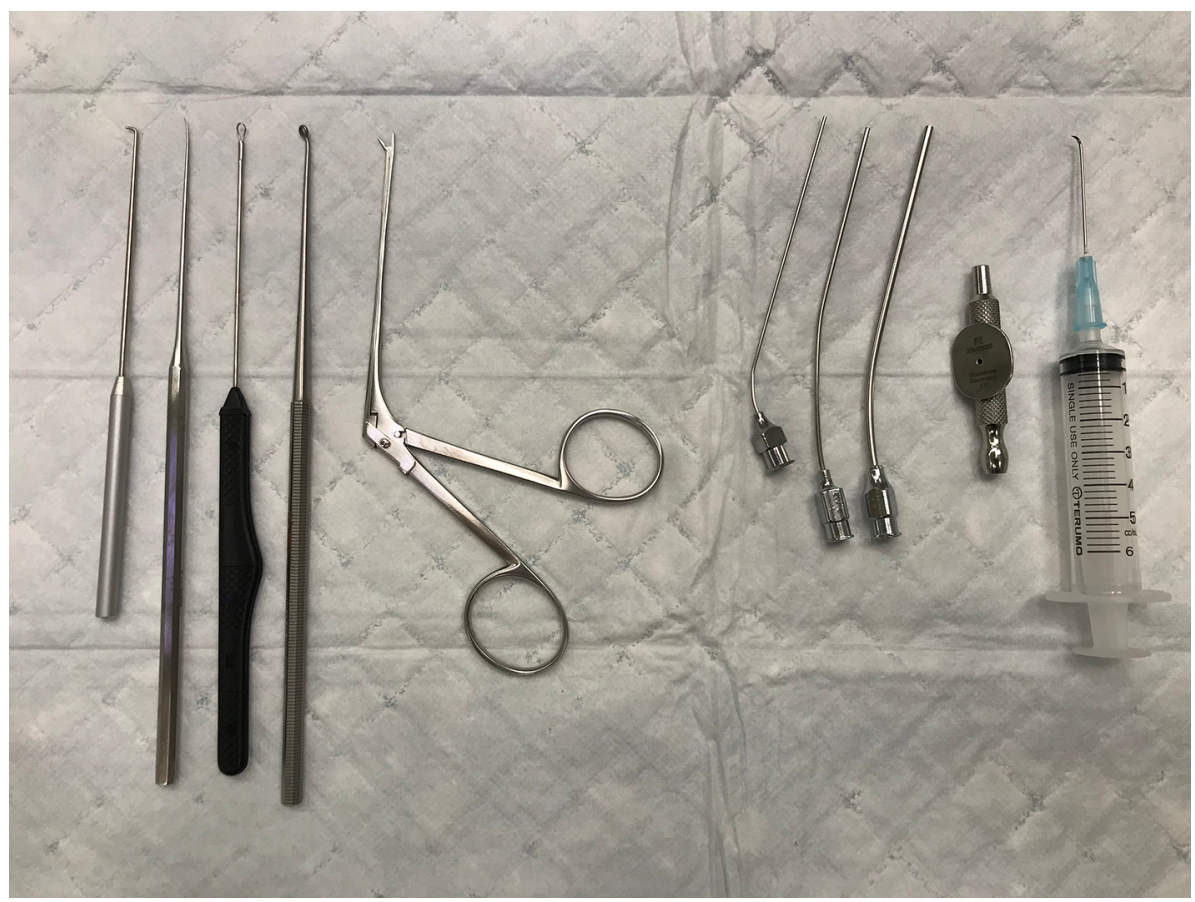

Figure I Instruments commonly used for removal of aural foreign body.

Note: From left to right: L-shaped hook, Rosen needle, wax curette, ring curette, crocodile forceps, three sets of microsuckers of varying sizes, sucker connecter with finger hole, and refashioned hypodermic needle.

not suitable for hard, spherical AFBs such as beads (unless there is a hole) or popcorn kernels, which are also among the commonly seen AFBs, due to the high risk of slippage and pushing the AFB deeper into the EAC. ${ }^{8}$

\section{L-shaped hook/right-angle hook}

This is shown in Figure 1.

\section{Technique}

The hook is passed beyond the $\mathrm{FB}$, rotated $90^{\circ}$, and used to coax the FB forward and out of the EAC.

\section{Good for}

This is good for non-graspable FBs (round or smooth objects).

\section{Note}

This method is only possible if the hook can be passed past the FB and should not be used in a struggling or non-cooperative child due to the risk of injuring the EAC with the hook.

\section{Refashioned hypodermic needle}

This is shown in Figure 1.

\section{Technique}

It is the same as that of L-shaped hook/right-angle hook.

\section{Good for}

This is good for non-graspable objects (round or smooth objects).

\section{Note}

This method can be employed in a non-otolaryngology setup, when an L-shaped hook/right-angle hook is not available. A syringe $(1,3,5$, or $10 \mathrm{~mL}$ can be used) is attached to a hypodermic needle (size 21, 22, or $23 \mathrm{G}$ ), and artery forceps are used to bend the needle distal tip to a right angle, turning it into an L-shaped hook. The syringe holding the bent needle is held in a pen-like fashion when extracting the AFB.

\section{Rosen needle/curved hook}

This is shown in Figure 1.

\section{Technique}

It is the same as that of L-shaped hook/right-angle hook.

\section{Good for}

This is good for non-graspable objects (round or smooth objects), beads with a hole in the center.

\section{Note}

It is useful when the space available to maneuver the L-shaped hook is too narrow. Ideal for beads with a hole in their center 
as the Rosen needle tip can be inserted into the hole to gently coax the FB out.

\section{Ring curette/wax curette/Jobson Horne probe}

This is shown in Figure 1.

\section{Technique}

Pass the ring curette beyond the FB and slowly draw it out of the EAC.

\section{Good for}

This is good for non-graspable objects (round or smooth objects).

\section{Note}

This is less traumatic than L-shaped hook.

\section{Lasso technique}

This is presented in Figure 2.

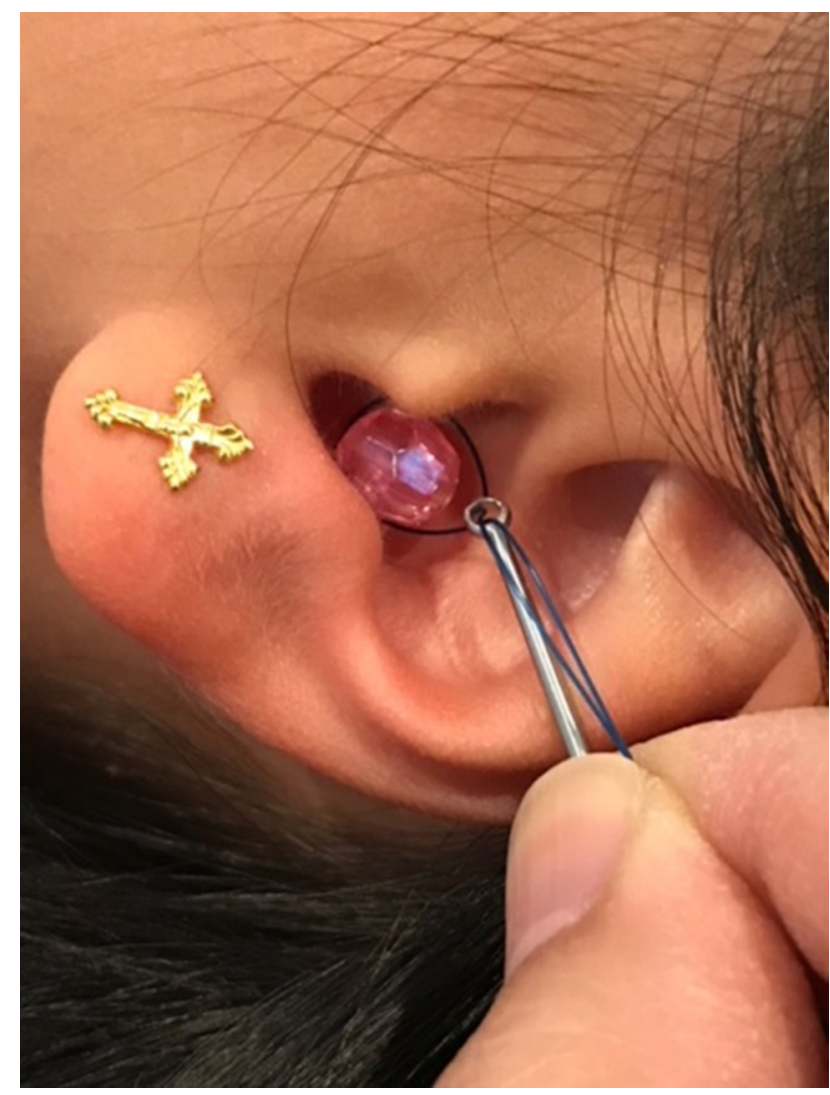

Figure 2 AFB being removed using the lasso technique. Abbreviation: AFB, aural foreign body.

\section{Technique}

A loop is created using a Jobson Horne probe/ring curette and a suture, and the loop lassos the FB and draws it out. Works best with monofilament non-absorbable synthetic suture material as this creates a strong, non-collapsing loop that can be easily positioned to lasso the $\mathrm{FB}^{9}$ (see Figure 3).

\section{Good for}

This is good for non-graspable objects (round or smooth objects), with barely any space to pass any rigid instrument beyond it.

\section{Note}

This is a favorable technique in children who are not tolerant of instruments touching the EAC and has the advantage of not pushing the FB further into the EAC. ${ }^{9}$

\section{Microsuction/ear toilet}

This is shown in Figure 1.

\section{Technique}

With the suction tip gently positioned next to the $\mathrm{FB}$, the finger hole is occluded to create more suction and remove the suction tip and FB as one.

\section{Good for}

This is good for spherical objects, friable materials, and dead insects.

\section{Note}

Warn patient about the loud noise and possibility of vertigo or giddiness post-suction. Also, there is a risk of trauma by accidentally injuring the EAC skin.

\section{Discussion}

AFB removal is technically more difficult and challenging compared to nasal FB removal because the outer ear is a complete canal, cartilaginous in the outer one third, and bony in the inner two thirds; thus, the space available to maneuver an instrument is very limited. In addition, the ear canal is supplied by a myriad of nerves, making it very sensitive. Each of the described methods has its benefits and disadvantages; hence, the selection of the method to remove AFB is to be made for each case, depending on the type and size of AFB and its location within the EAC. Ideally, all AFBs should be removed using an ENT operating microscope as it provides excellent illumination and magnification. In the absence of 


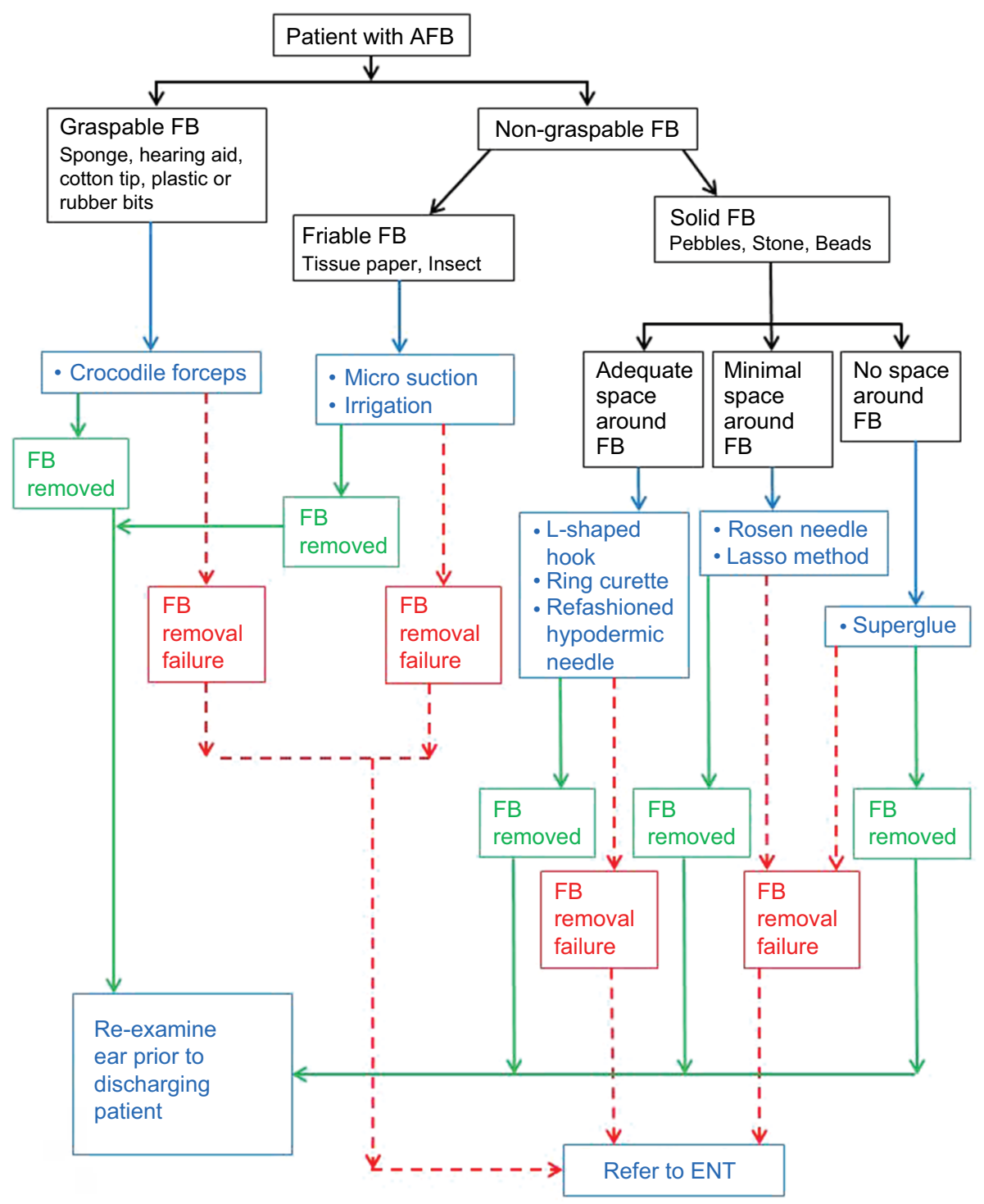

Figure 3 Flowchart describing what to do when presented with an AFB.

Note: The aim is to reduce the number of attempts, preferably to achieve success on the first try as often as possible.

Abbreviations: AFB, aural foreign body; FB, foreign body.

a microscope, a good headlight with appropriate-sized aural speculum can be used instead. If trauma is caused to the ear, prophylaxis against otitis externa should be provided with topical antibiotic ear drops. Only fluoroquinolone antibiotic drops should be used in cases with TM perforation due to risk of ototoxicity with other antibiotic drops. See Figure 3 for a flowchart to aid in selecting the right method for AFB removal.

\section{Conclusion}

Since AFBs are difficult to remove, and as patients normally permit only a few attempts due to pain, ideally, the first attempt should be the only attempt. All medical personnel to be consulted should be aware of the most suitable instruments for the various types of AFB, in order to reduce number of removal attempts. In the event of a single failed attempt or if failure to remove the AFB is anticipated, early referral for an ENT consultation is strongly recommended.

\section{Acknowledgments}

I would like to thank Dr Vicky Tobin, Professor Warren Rozen, and Frankston Hospital Library.

This project was not financially supported by any external sources and was fully funded by the Department of Surgery, Frankston Hospital. 


\section{Disclosure}

The author reports no conflicts of interest in this work.

\section{References}

1. Ng TT, Lim JWJ. A 5 year review of aural foreign body removal in a major Victorian hospital. Aust J Otolaryngol. 2018;1:25.

2. Davies PH, Benger JR. Foreign bodies in the nose and ear: a review of techniques for removal in the emergency department. J Accid Emerg Med. 2000;17(2):91-94.

3. Anusha B, Purushotman R, Lina LC, Avatar S. Superglue accidentally used as ear drops. Med J Malaysia. 2012;67(2):212-213.

4. Dimitriadis PA, Rourke T, Colquhoun-Flannery W, Herdman R, Corbridge RJ. Superglue ear: our experience and a review of the literature. $B-E N T$. 2013;9(4):325-328.
5. Friedman EM. Removal of foreign bodies from the ear and nose. $N E n g l$ J Med Overseas Ed. 2016;374(7):e7.

6. Kumar S, Kumar M, Lesser T, Banhegyi G. Foreign bodies in the ear: a simple technique for removal analysed in vitro. Emerg Med $J$. 2005;22(4):266-268.

7. Kumar S. Management of foreign bodies in the ear, nose and throat. Emerg Med Australas. 2004;16(1):17-20.

8. Kadish H. Ear and nose foreign bodies: "it is all about the tools". Clin Pediatr (Phila). 2005;44(8):665-670.

9. Crockett A, Haslegrave C, Trinidade A, Andreou Z, Kothari P. Removal of foreign body from the external ear using the lasso technique. Clin Otolaryngol. 2011;36(2):194.
Open Access Emergency Medicine

\section{Publish your work in this journal}

The Open Access Emergency Medicine is an international, peerreviewed, open access journal publishing original research, reports, editorials, reviews and commentaries on all aspects of emergency medicine. The manuscript management system is completely online and includes a very quick and fair peer-review system, which is all
Dovepress

easy to use. Visit http://www.dovepress.com/testimonials.php to read real quotes from published authors. 